\title{
Generalized Anxiety Disorder and Its Associated Factors Among Health Care Workers Fighting COVID-19 in Southern Ethiopia
}

This article was published in the following Dove Press journal: Psychology Research and Behavior Management

\author{
Abinet Teshome' \\ Mustefa Glagn ${ }^{2}$ \\ Mulugeta Shegaze $\mathbb{D D}^{2}$ \\ Beemnet Tekabe ${ }^{2}$ \\ Asmare Getie ${ }^{3}$ \\ Genet Assefa ${ }^{3}$ \\ Dinkalem Getahun (D) ${ }^{3}$ \\ Tesfaye Kanko' \\ Tamiru Getachew $\mathbb{I D}^{4}$ \\ Nuhamin Yenesew ${ }^{5}$ \\ Zebene Temtmie ${ }^{5}$ \\ Kabtamu Tolosie ${ }^{6}$ \\ 'Department of Biomedical Science, \\ School of Medicine, Arba Minch \\ University, Arba Minch, Ethiopia; ${ }^{2}$ School \\ of Public Health, College of Medicine and \\ Health Science, Arba Minch University, \\ Arba Minch, Ethiopia; ${ }^{3}$ School of Nursing, \\ Arba Minch University, Arba Minch, \\ Ethiopia; ${ }^{4}$ Department of Anatomy, Arba \\ Minch University, Arba Minch, Ethiopia; \\ ${ }^{5}$ Department of Psychology, Arba Minch \\ University, Arba Minch, Ethiopia; \\ ${ }^{6}$ Department of Statistics, Arba Minch \\ University, Arba Minch, Ethiopia
}

Background: Healthcare workers (HCWs) are among the many groups of people who are in the frontline caring for people and facing heavy workloads, life-or-death decisions, risk of infection, and have been facing various psychosocial problems. So, monitoring mental health issues to understand the mediating factors and inform evidence-based interventions in a timely fashion is vital.

Purpose: This study aimed to assess generalized anxiety disorder and its associated factors among HCWs fighting COVID-19 in Southern Ethiopia.

Patients and Methods: An institution-based cross-sectional study was conducted among $798 \mathrm{HCWs}$ from 20 May to 20 June 2020. A pre-tested and structured intervieweradministered KOBO collect survey tool was used to collect data. The study participants were selected using a simple random sampling technique by allocating a proportion to each health institute. The association between the level of generalized anxiety disorder and its independent variables was examined by ordinal logistic regression. Assumptions for the proportional odds model were checked using parallel line tests. An adjusted proportional odds ratio with a $95 \%$ CI was used to calculate the strength of the statistical association between the independent and dependent variables.

Results: The prevalence of mild and moderate anxiety disorder among HCWs was $29.3 \%$ and $6.3 \%$, respectively. Contact with confirmed or suspected cases (aPOR $=1.97 ; 95 \% \mathrm{CI}$ : $1.239,3.132$ ), no COVID-19 updates ( $\mathrm{aPOR}=4.816,95 \% \mathrm{CI}=2.957,7.842$ ), no confidence on coping with stresses (aPOR $=2.74,95 \% \mathrm{CI}=1.633$, 4.606), and COVID-19-related worry ( $\mathrm{aPOR}=1.85,95 \% \mathrm{CI}=1.120,3.056)$ were positively associated with higher-order anxiety disorder. However, not feeling overwhelmed by the demands of everyday life ( $\mathrm{aPOR}=0.52$, $95 \% \mathrm{CI}=0.370,0.733)$ and feeling cannot make it ( $\mathrm{aPOR}=0.44,95 \% \mathrm{CI}=0.308,0.626)$ were negatively associated with a higher order of anxiety.

Conclusion: The study revealed that the prevalence of anxiety disorder among HCWs was high in the study area. The findings of the current study suggest immediate psychological intervention for health care workers in the study area is vital. Therefore, proactive measures should be taken by the stakeholders at different hierarchies to promote the psychological wellbeing of HCWs in order to control the impact of the pandemic on the HCWs, and containing the pandemic.

Keywords: anxiety, health care workers, associated factor, Ethiopia

\section{Introduction}

Health care workers (HCWs) who are in the frontline caring for people with COVID-19 infection have been facing various psychosocial problems, including a high risk of infection and inadequate protection from contamination, overwork,
School of Public Health, College of

Medicine and Health Science, Arba Minch

University, P.O. Box 21, Arba Minch,

Ethiopia

Tel +251913976776

Email mustesami02@gmail.com
Psychology Research and Behavior Management 2020:13 907-917

907

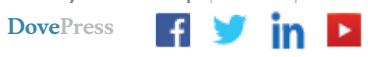

http://doi.org/| 0.21 47/PRBM.S282822 
frustration, a lack of contact with their families, and loved ones. The severe situation is causing mental health problems such as anxiety. ${ }^{1-3}$ Generalized anxiety disorder is the most frequently occurring mental health disorder, which is characterized by excessive worry or fearfulness about events and is associated with heightened tension, nervousness, and irritability; it may cause physical symptoms such as restlessness, fatigue, muscle stiffness, and trouble concentrating or sleeping. ${ }^{4,5}$ The HCWs are facing the pressure of working in resource-deprived settings and ever-growing patient load all over the world. ${ }^{6}$

According to the studies conducted in the era of Severe Acute Respiratory Syndrome (SARS) and Ebola epidemics, the onset of a sudden and immediately lifethreatening illness could lead to extraordinary amounts of pressure on HCWs and might cause adverse psychological disorders, such as anxiety, fear, and stigmatization. ${ }^{7-10}$ The other study conducted in Italy reported a significant proportion of HCWs experienced anxiety, depression and sleep disturbances during COVID-19. ${ }^{11}$ This psychological pressure exerts an adverse effect on the quality of care given for patients. HCWs are expected to wear heavy protective garments and other personal protective equipment during pandemic like COVID-19, making it much more difficult to carry out medical operations or procedures than under normal conditions. These factors, together with the fear of being contagious and infecting others, physical exhaustion, inadequate personal equipment, and the need to make ethically difficult decisions on the rationing of care could increase the possibility of psychological issues among HCWs. ${ }^{9,12-14}$ A study conducted in China reported that $13 \%$ of HCWs experienced anxiety during the fight against COVID-19. ${ }^{15}$ Another study conducted on the psychological impact of the COVID-19 pandemic on health care workers in Singapore reported a $10.8 \%$ anxiety prevalence. ${ }^{16}$ Besides, a Hong Kong study found that health workers suffered high anxiety scores after directly treating confirmed SARS patients. ${ }^{17}$

Studies revealed that having comorbidities, living in the rural areas, being a female health worker, marital status, profession, having children, and contact with COVID-19 patients were the most common risk factors for developing anxiety among health workers. ${ }^{15,16}$

Ethiopia faces the most critical phase of the pandemic, with community transmission. HCWs across the country are facing a fight like never before. Frontline health-care workers, faced with heavy workloads, life-or-death decisions, and risk of infection, are particularly affected. ${ }^{18}$ Even though there are some studies conducted on the psychosocial impact of COVID-19 and its associated factors globally, it is a new disease and the medical system and culture of different countries vary which necessitates the need for further research on the psychological experience of frontline health workers fighting against COVID-19. Currently, as far as our literature search is concerned, there are no published studies on anxiety related to COVID-19 in Ethiopia in general and Southern region in particular. Therefore, this study aimed to assess generalized anxiety disorder and its associated factors among healthcare workers fighting COVID-19 in Southern Ethiopia. The findings of this study are timely and would help the local program planners and policymakers to plan appropriate interventions at the early stage to prevent a detrimental psychological outcome of HCWs.

\section{Methods and Materials}

\section{Study Settings, Design, and Population}

Institution-based cross-sectional study was conducted among health workers working in Gamo, Gofa, Konso, and South Omo Zones of Southern Ethiopia from 20, May to 20, June 2020.

There are a total of two general and 10 primary hospitals, and 134 health centers in the four zones. There are 4820 health professionals providing health services in the Zones. All health care workers working in four Zones during the data collection period were included in the study.

\section{Sample Size Estimation}

A single population proportion formula $\left((\mathrm{Z} \alpha / 2) \mathrm{pq} / \mathrm{d}^{2}\right)$ was used to estimate the sample size required for the study. The sample size calculation assumed the proportion (p), the estimated level of anxiety among HCWs was estimated to be $50 \%$ because there is no prior study finding in Ethiopia, 95\% confidence level, margin of error of 5\%, and a design effect of 2 which gave the sample size of 768 . In consideration of a $10 \%$ non-response rate, the final sample size was $845 \mathrm{HCWs}$. Mathematically, it is calculated as follows:

$$
\begin{aligned}
& n=\left((Z \alpha / 2) p q / d^{2}\right)=\left((1.96)^{2} * 0.5 *(1-0.5)\right)=384 \\
& (0.05)^{2}
\end{aligned}
$$

Where: $n=$ the required sample size 
$\mathrm{Z}=$ the standard score corresponding $95 \%$ confidence interval (1.96)

$\mathrm{P}=$ level of anxiety among HCWs, it was estimated to be $50 \%$

$\mathrm{d}=$ margin of error, $5 \%$

By considering a design effect of 2 because we used a multi-stage sampling technique $(384 * 2=768)$. Then, we considered a $10 \%$ non-response rate, the final sample size used for this study was $768+77=845$.

\section{Sampling Procedure}

The study participants were selected using a multistage sampling technique. First, 20\% of health institutions were selected using a simple random sampling technique (computer-generated random numbers) after allocating a proportion to each Zone based on the size of health institutions. Then, the sample size was proportionally allocated to the health institutes based on the size of health care workers. Lists of active health care workers were taken from each selected health institute. Finally, a simple random sampling technique (computergenerated random number) was implemented to recruit the health professionals in each selected health institute.

\section{Data Collection Procedure and Measurement}

A pretested and structured interviewer-administered questionnaire was used to collect data. The tools were developed by reviewing different works of literature and the World Health Organization (WHO) guidelines. The survey questionnaire included Socio-Demographic characteristics, medical history, alcohol use, physical exercise, and miscellaneous psychosocial questions. Generalized Anxiety Disorder Scale (GAD-7) was used to assess the level of anxiety among health professionals. ${ }^{19}$ It is a 7 -item questionnaire developed to screen patients for anxiety and rate the severity of anxiety. Each item is rated on a 4-point likert-scale ranging from 0 (not at all) to 3 (nearly every day) on the symptoms in the previous 2 weeks. The total score of GAD-7 ranged from 0 to 21 , with increasing scores indicated more severe functional impairments as a result of anxiety. For the purpose of this study, the score of anxiety assessing questions was calculated for each respondent then the overall score was computed and the levels of anxiety were classified into none to minimal, mild, moderate, and severe. Scores represent $0-5$ None to Minimal, 6-10 mild, 11-15 moderate, and 15-21 severe anxiety. During data collection, a reliability analysis was done and the result showed a good score of internal consistency between the items (Cronbach's alpha $=0.807$ ). Language experts translated the questionnaire from English to Amharic and back to English to ensure consistency in meaning. A pretest was conducted on an unselected health institute by taking $5 \%$ of the total sample size. After we made appropriate corrections, the revised version of the questionnaire was used for final data collection. Twelve trained public health professionals participated in the data collection. We used KOBO collect survey tool to collect data. KOBO collect survey tool is mobile applications that allow for the collection of data using mobile devices, analysis of data, and storage of data - either online or offline. Data were collected using face-to-face data collection technique. All WHO recommended COVID-19 protective measures were applied during the data collection period.

\section{Data Quality Management}

Data collectors and supervisors were provided with intensive training on the techniques of data collection and components of the instrument. Before the commencement of the data collection, a pretest was conducted. A standard tool, which was developed by experts, was used to collect the information. The KOBO collect survey tool that was very important to control the quality of data was used to collect data by using tablets. The Authors and supervisors critically checked the data for completeness before being uploaded to the KOBO collect cloud server.

\section{Statistical Analysis}

The collected data were downloaded from the KOBO collect. It was then edited and cleaned for inconsistencies, missing values using excel, and then exported to SPSS version 25 (SPSS Inc., Chicago, IL, USA) for further analysis. Descriptive statistics were computed and summarized in tables, figures, and text with frequencies, mean, or standard deviations where appropriate. The association between the level of generalized anxiety disorder and its independent variables was examined by ordinal logistic regression. The ordinal logistic regression model was used because the level of anxiety was determined by using ordinal data (None to Minimal, mild, moderate, and severe). The proportional odds model (POM) was fitted to identify factors associated with the level of generalized anxiety disorder. The necessary assumptions for POM were checked using parallel line tests. The 
chi-squared test $\left(\chi^{2}\right)$ for the proportional odds assumption was calculated to see whether the model assumptions were violated or not. The Pearson $\chi 2$ goodness-of-fit test showed that the observed data were consistent with the fitted model; the deviance test ( $\chi 2=920.875, p=0.622$ ) was non-significant. Additionally, the appropriateness of the POM was calculated by the parallel line test, and it showed that the general model did not significantly differ from the fitted POM ( $p=0.092)$, this indicated that the model fit the data well. Furthermore, the $\chi 2$ test of parallelism showed that the odds ratios were constant across all cutoff points of anxiety level for the final model at a 5\% level of significance. Bivariable analyses were performed between the dependent and independent variables. All variables with a $p$-value $<0.25$ in the bivariable analysis were fitted into the multivariable POM to control for confounding effects. An adjusted proportional odds ratio with a 95\% CI was used to calculate the strength of the statistical association between the independent and dependent variables.

\section{Ethical Consideration}

Ethical approval and clearance were obtained from Arba Minch University Institutional research Ethics Review Board, College of Medicine and Health Sciences with reference number (IRB/406/12). A letter of cooperation was obtained from the Zonal health department and health institutes of the respective Zones. The purpose of the study was explained and informed written consent was taken from each health care worker. To ensure confidentiality, their names, and other personal identifiers were not registered in the survey tool. Besides, this study was conducted in accordance with the Declaration of Helsinki, and all ethical and professional considerations were followed throughout the study to keep participants' data strictly confidential.

\section{Results}

\section{Socio-Demographic Characteristics and Medical History of Participants}

The data were collected from 798 study participants with a $94.2 \%$ response rate. Out of the total respondents, 482 $(60.4 \%)$ were male. The mean age $( \pm \mathrm{SD})$ of the participants was 29.29 years $( \pm 5.69)$ years. The majority of the participants were town residents $(88.3 \%)$ and more than half of the participants were protestant religion followers (52.3). Among the participants' health workers, 356
(44.6\%) were clinical nurses and 363 (45.5\%) were diploma holders (Table 1).

\section{Individual Psychosocial Factors and Substance Use of Health Care Workers}

Among the respondents, $85 \%$ of them reported no adequate personal protective equipment in their health institute. The majority of the participants (96\%) had access to media. Of respondents, 96 (12\%) had no confidence in coping with stress-related with COVID-19, 682 (85.5\%) had a feeling of susceptibility, and 633 (79.3\%) had COVID-19 related worries. Among the participants, 630 (78.9\%) believed that the suggested prevention and control practices can contain the pandemic (Table 2).

\section{Family, Community, and Government Support}

Among health care workers who participated in this study, $31.0 \%, 51.2 \%, 16.2 \%$ and $1.6 \%$ of them believed the community members were responding to the prevention practice of COVID-19, not at all, somewhat, moderately, and to the great extent, respectively. More than two-thirds $(72.2 \%)$ of HCWs felt valued by their families. The data revealed that from the participant health care workers, $27.9 \%, 51.4 \%, 17.7 \%$ and $3.0 \%$ of them agreed the government is supporting the prevention and control of COVID-19, not at all, somewhat, moderately, and to the great extent, respectively.

\section{Prevalence of Anxiety Disorder}

The prevalence of mild and moderate anxiety disorder among HCWs was $29.3 \%$ and $6.3 \%$, respectively. There was no severe stage of anxiety observed during the data collection period (Figure 1).

\section{Factors Associated with Anxiety Disorder Among Healthcare Workers}

In ordinal logistic regression analysis, variables such as contact with confirmed or suspected cases, COVID-19 updates, confidence in coping with stresses, COVID-19related worries, feel overwhelmed by the demands of everyday life, and feeling cannot make it were statistically significantly associated with an anxiety disorder. The risk of being in the higher order of anxiety was almost two times (aPOR $=1.97 ; 95 \%$ CI: 1.239, 3.132) higher among health professionals who had contact with confirmed or suspected COVID-19 cases compared to who had no 
Table I Socio-Demographic and Medical History of HCWs Working in Response to COVID-19 in Southern Ethiopia from 20 , May to 20 , June 2020

\begin{tabular}{|c|c|c|}
\hline Variables & Frequency $(\mathrm{N}=\mathbf{7 9 8})$ & Percentage \\
\hline \multicolumn{3}{|l|}{ Sex } \\
\hline Female & 316 & 39.6 \\
\hline Male & 482 & 60.4 \\
\hline \multicolumn{3}{|l|}{ Marital status } \\
\hline Single & 306 & 38.2 \\
\hline Married & 492 & 61.7 \\
\hline \multicolumn{3}{|l|}{ Having children } \\
\hline No & 353 & 44.2 \\
\hline yes & 445 & 55.8 \\
\hline \multicolumn{3}{|l|}{ Resident } \\
\hline Rural & 93 & 11.7 \\
\hline Town & 705 & 88.3 \\
\hline \multicolumn{3}{|l|}{ Living arrangement } \\
\hline Alone & 239 & 29.9 \\
\hline With friends & 20 & 2.5 \\
\hline With parents & 161 & 20.2 \\
\hline With spouse & 378 & 47.4 \\
\hline \multicolumn{3}{|l|}{ Profession } \\
\hline Clinical Nurse & 356 & 44.6 \\
\hline Medical Doctor & 65 & 8.1 \\
\hline Medical laboratory & 84 & 10.5 \\
\hline Midwifery & 120 & 15.0 \\
\hline Pharmacist & 77 & 9.6 \\
\hline Public health officer & 96 & 12.0 \\
\hline \multicolumn{3}{|l|}{ Religion } \\
\hline Muslim & 38 & 4.8 \\
\hline Orthodox & 338 & 42.4 \\
\hline Protestant & 417 & 52.3 \\
\hline Other* & 5 & 0.6 \\
\hline \multicolumn{3}{|l|}{ Education status } \\
\hline Diploma & 363 & 45.5 \\
\hline First degree & 359 & 45.0 \\
\hline Level 3 or 4 & 60 & 7.5 \\
\hline Second degree & 16 & 2.0 \\
\hline \multicolumn{3}{|l|}{ Ward/case team } \\
\hline Dispensary & 61 & 7.6 \\
\hline Emergency ward & 98 & 12.3 \\
\hline Laboratory & 67 & 8.4 \\
\hline Maternity (Gyn/Obs) ward & 160 & 20.1 \\
\hline Medical ward & 98 & 12.3 \\
\hline OPD (including paramedical) & 187 & 23.4 \\
\hline Pediatrics (including NICU) ward & 66 & 8.3 \\
\hline Surgical (including OR) ward & 61 & 7.6 \\
\hline \multicolumn{3}{|l|}{ Medical Conditions/Comorbidity } \\
\hline yes & 44 & 5.5 \\
\hline No & 754 & 94.5 \\
\hline
\end{tabular}

Notes: *Atheist, Adventist, and Catholic

Abbreviations: Gyn/obs, gyenocology/obstetric; NICU, neonatal intensive care unit; OPD, outpatient department; OR, operation room. contact. Likewise, health care professionals who had no COVID-19 update were almost 5 times (aPOR $=4.81,95 \%$ $\mathrm{CI}=2.957,7.842$ ) more likely to develop a higher order of anxiety than those HCWs who had an update. Similarly, HCWs who were somewhat confident in coping with stress were 2.7 times $(\mathrm{aPOR}=2.74,95 \% \mathrm{CI}=1.633,4.606)$ more likely to develop a higher order of anxiety disorder than their counterparts. Regarding COVID-19 related worries, the odds of health care workers who had COVID-19 related worries were 1.85 times $(\mathrm{aPOR}=1.85,95 \%$ $\mathrm{CI}=1.120,3.056)$ more likely to experience higher order of anxiety than who did not worry. The odds of health care workers who had not felt overwhelmed by the demands of everyday life were $48 \%$ (aPOR $=0.52,95 \%$ $\mathrm{CI}=0.370,0.733$ ) less likely to develop a higher order of anxiety than who was feeling overwhelmed. Similarly, the odds of health care professionals who did not feel that they cannot make it were also 56\% (aPOR $=0.44,95 \%$ $\mathrm{CI}=0.308,0.626$ ) less likely to experience higher order of anxiety than who felt they can make it (Table 3).

\section{Discussion}

The study examined the prevalence of anxiety among health care workers and identified risk factors for increased anxiety. The finding of this study revealed that $29.3 \%$ and $6.3 \%$ of HCWs suffered from mild and moderate anxiety disorder, respectively. A study from China reported that $44.6 \%$ of the HCWs had symptoms of anxiety. ${ }^{20}$ A related study conducted in Turkey indicated that $51.6 \%$ of respondents had anxiety-related symptoms. Of this, $16.3,13.1 \%, 10.6 \%$, and $11.5 \%$, of HCWs had mild, moderate, severe, and extremely severe anxiety symptoms, respectively. ${ }^{21}$ Another recent meta-analysis of studies reported the prevalence rate of the anxiety of HCWs during COVID-19 was ranging between 22.6\%$36.3 \% .{ }^{22}$ Pockets of studies from different corners of the world had been reported the prevalence of anxiety among HCWs with a range of $11.350 \%{ }^{20,23-25}$ and finding of the present study was within this reported range. The finding of the current study showed that the majority of the HCWs experienced mild symptoms of anxiety, while moderate and severe symptoms were less common among the participants. In our view the reported figure is huge since the study was conducted at the early stage of the pandemic in Ethiopia; this alarms the need for early detection and the importance of picking up and effectively treating the milder clinical symptoms of anxiety 
Table 2 Individual Psycho-Social Factors and Substance Use of Health Care Workers, Working in Response to COVID-19 in Southern Ethiopia from 20, May to 20, June 2020

\begin{tabular}{|c|c|c|}
\hline Variables & $\begin{array}{l}\text { Frequency } \\
(\mathrm{N}=798)\end{array}$ & Percentage \\
\hline \multicolumn{3}{|l|}{$\begin{array}{l}\text { Contact with confirmed or suspected } \\
\text { cases }\end{array}$} \\
\hline No & 688 & 86.2 \\
\hline Yes & 110 & 13.8 \\
\hline \multicolumn{3}{|l|}{ Type of media } \\
\hline Social media only & 76 & 9.5 \\
\hline Mainstream media only & 121 & 15.2 \\
\hline Both & 569 & 71.3 \\
\hline No & 32 & 4.0 \\
\hline \multicolumn{3}{|l|}{ COVID-19 updates } \\
\hline No & 105 & 13.2 \\
\hline Yes & 693 & 86.8 \\
\hline \multicolumn{3}{|l|}{ Confident in coping with stresses } \\
\hline Not at all & 96 & 12.0 \\
\hline Somewhat & 261 & 32.7 \\
\hline Moderately & 307 & 38.5 \\
\hline To a great extent & 134 & 16.8 \\
\hline \multicolumn{3}{|l|}{ Drink alcohol } \\
\hline No & 632 & 79.2 \\
\hline Yes & 166 & 20.8 \\
\hline \multicolumn{3}{|l|}{ Smoke cigarette } \\
\hline No & 790 & 99.0 \\
\hline Yes & 8 & 1.0 \\
\hline \multicolumn{3}{|l|}{ Physical exercise } \\
\hline No & 335 & 42.0 \\
\hline Yes & 463 & 58.0 \\
\hline \multicolumn{3}{|l|}{ Have anyone can trust and confide in } \\
\hline No & 124 & 15.5 \\
\hline Yes & 674 & 84.5 \\
\hline \multicolumn{3}{|l|}{ Get along well with people } \\
\hline No & 54 & 6.8 \\
\hline Yes & 744 & 93.2 \\
\hline \multicolumn{3}{|l|}{$\begin{array}{l}\text { Feel overwhelmed by the demands of } \\
\text { everyday life }\end{array}$} \\
\hline No & 359 & 45.0 \\
\hline Yes & & \\
\hline \multicolumn{3}{|l|}{ Feel you cannot make it } \\
\hline No & 572 & 71.7 \\
\hline Yes & 226 & 28.3 \\
\hline \multicolumn{3}{|l|}{ Influenced by people with strong opinions } \\
\hline No & 552 & 69.2 \\
\hline Yes & 246 & 30.8 \\
\hline \multicolumn{3}{|l|}{ Worry about what other people think of you } \\
\hline No & 649 & 81.3 \\
\hline Yes & 149 & 18.7 \\
\hline
\end{tabular}

before they evolve to more complex psychological disorders. Furthermore, a lower moderate and severe level of anxiety might be associated with the lower report of confirmed cases due to limited testing capacity during the study period in comparison with other countries. There was also a lower report of confirmed cases in the study area. A study from China which was the epicenter of COVID-19 showed that working outside the epicenter was associated with a lower risk of psychological symptoms than working in the epicenter even in the same country. ${ }^{20}$ However, mild anxiety symptoms were higher among HCWs in the study area. This high level of anxiety among the HCWs in our study area could be attributed to fear of transmission of the disease to their family, no updates on COVID-19-related issues especially HCWs working in a rural area with no mobile network and internet connection, lack of specific drugs treating COVID-19 patients, an increasing number of suspected and confirmed cases and inadequate personal protective gears. Eighty-five percent of the participants perceived that personal protective equipment was inadequate in their health institutions. The difference in the prevalence of anxiety in different parts of the world could be attributed to variations in the health system characteristics, the culture of patient care, technologies, availability of PPE, and the tool used to assess anxiety and heterogeneity in cut off points. In the present study, we used the standard and validated tool developed for measuring generalized anxiety disorder after we checked its validity and reliability in our context. ${ }^{19}$

Authorities at different hierarchies should assess the hazards to which their health care workers are exposed; evaluate the risk of exposure; and select, implement, and ensure workers use controls to prevent exposure to the virus. Occupational health surveillance has paramount importance to enhance the wellbeing of HCWs. Such type of study is helpful for different stakeholders to monitor mental health issues to understand the mediating factors and inform evidence-based interventions in a timely fashion. $^{26,27}$

Our study further indicated that health professionals who had contact with confirmed or suspected COVID-19 cases were two times more likely to develop a higher order of anxiety than those who had no contact. The finding of this study was consistent with the studies conducted in China, ${ }^{24,25}$ the score of anxiety increases among health 


\section{Level of anxiety}

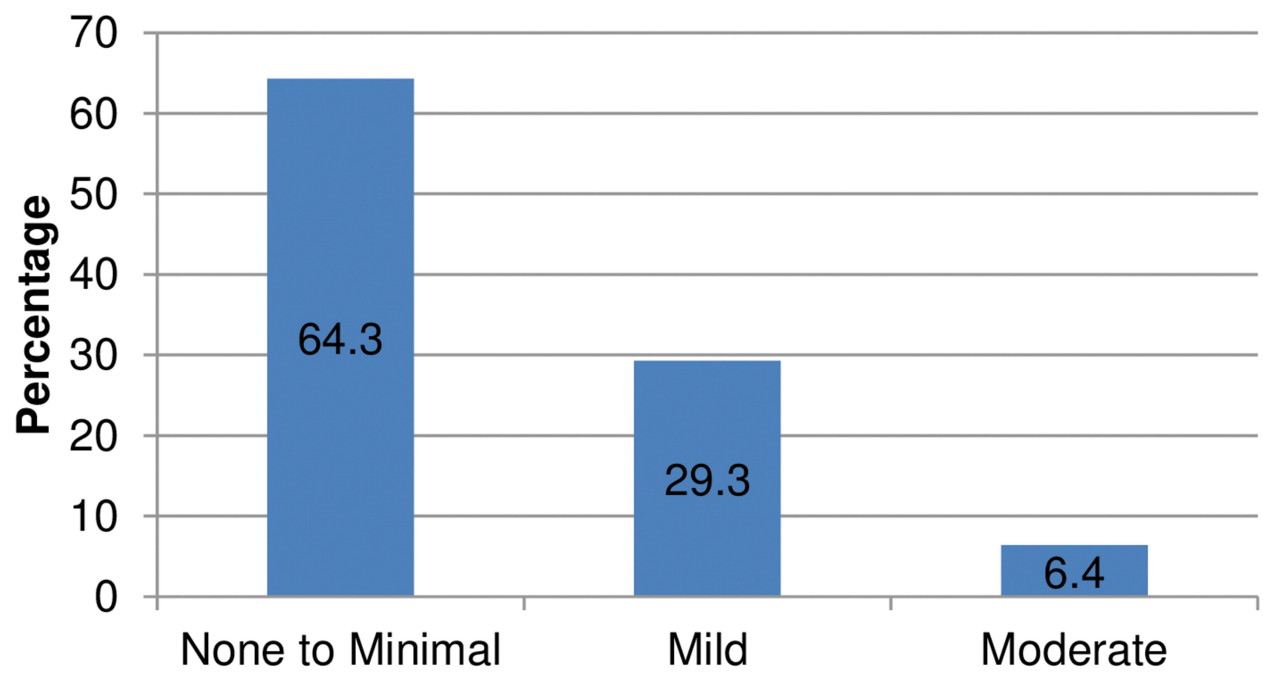

Figure I Level of generalized anxiety disorder of HCWs working in response to COVID-19 in Southern Ethiopia from 20, May to 20, June 2020.

care workers having direct contact with suspected and confirmed cases. This can be reasoned out that contact with suspected or confirmed cases increases the risk of transmission and exposure. The health workers working in low resource settings (weak health system, low economic status, and poor technology) like Ethiopia may limit their ability to follow the recommended measures; these and other factors exacerbate the fear of HCWs. Although the Ethiopian government and people presented recognition for HCWs for their dedication in fighting COVID-19 which could make health workers feel honored and proud to participate in this difficult assignment - the local and national authorities should also focus on implementing measures targeting the psychological well-being of HCWs.

Likewise, health care professionals who had no COVID-19 update were almost 5 times more likely to develop a higher order of anxiety than those health care professionals who had an update. This can be explained by HCWs getting information on the route of transmission, the availability and effectiveness of medicines/vaccines, experience in handling COVID-19 patient, the number of infected cases and locations, and advice on prevention of the COVID-19 increase the confidence of $\mathrm{HCWs}$ otherwise working in an uncertain environment without up to date information might worsen anxiety. This finding was also in agreement with the study conducted in China. ${ }^{29}$ The odds of health care professionals who had COVID-19 related worry were more likely to experience higher order of anxiety than those health care workers who did not worry. This might be justified as health care workers are working in a highly risky environment so that increases the probability of being infected so that they might worry about transmitting the infection to their families and loved ones, separating with their children, and being stigmatized. The finding was also supported by other studies, ${ }^{28,29}$ respondents were very worried or somewhat worried about other family members getting COVID-19. Furthermore, personal protective equipment, such as surgical masks face mask, eye goggles, protective clothing, gloves, and sanitizers, were severely deficient during the early stages of the outbreak in Ethiopia which might exacerbate worries of health care workers. Overwhelmed by daily activities and cannot make it were also significantly associated with developing higher order of anxiety among HCWs. This might be due to work overload, inner conflict about competing needs and demands of HCWs, stigma, and fear of being removed from their duties during a crisis heighten anxiety.

\section{Limitation of the Study}

This study has some limitations which have to be taken into consideration while interpreting the findings. As being crosssectional in the design, it does not confirm the definitive cause and effect relationship. We were unable to differentiate the preexisting anxiety from the new cases of anxiety. The other limitation of the study might be social desirability bias but we tried to minimize it by reminding participants about confidentiality and anonymity during data collection. However, the current study had strength; To the best of our knowledge, this is the first study assessed the prevalence of anxiety among HCWs during COVID-19 in Ethiopia, we used a strong method of analysis (Ordinal logistic regression), a validated and standardized tool with an appropriate cut off points to 


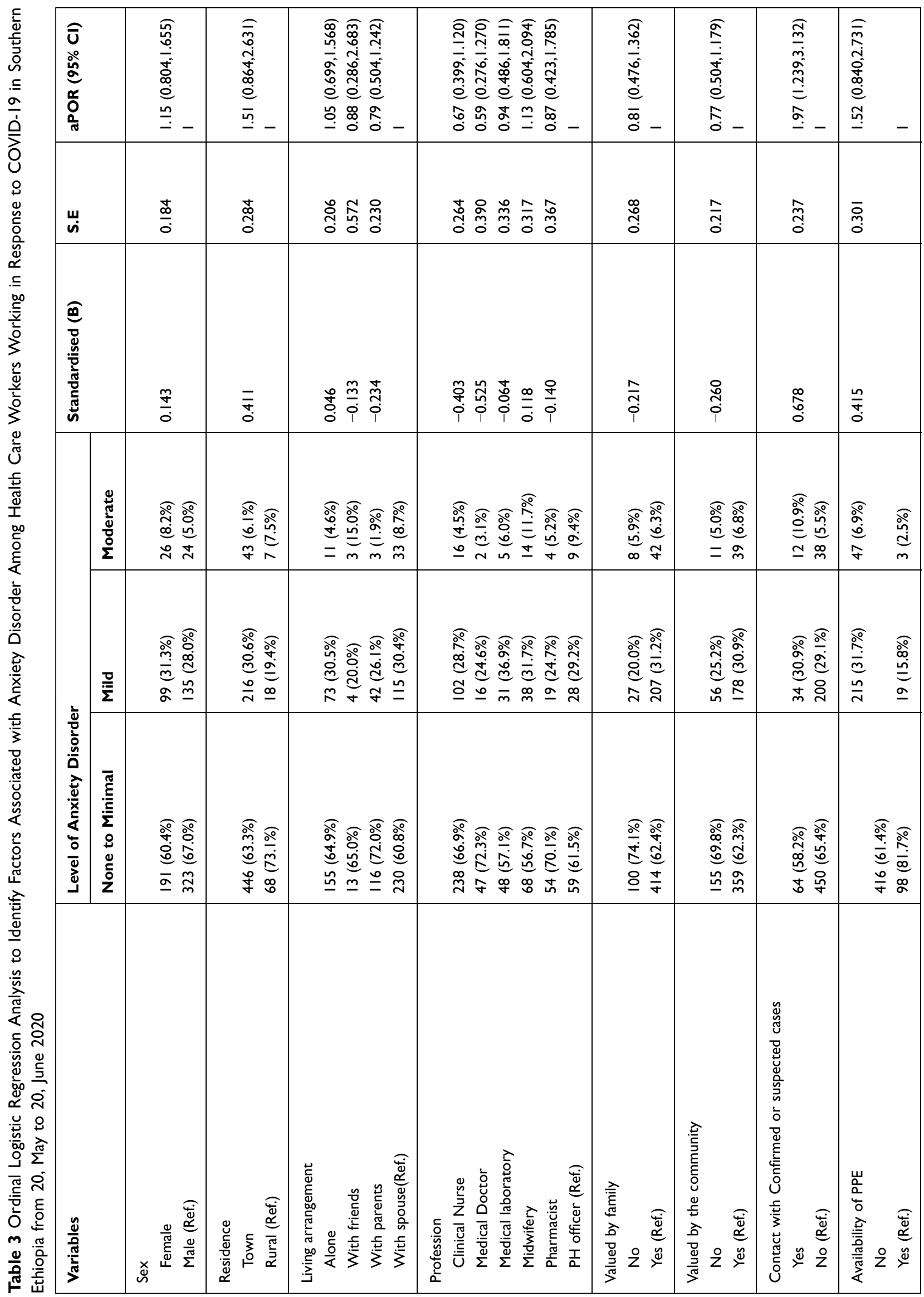




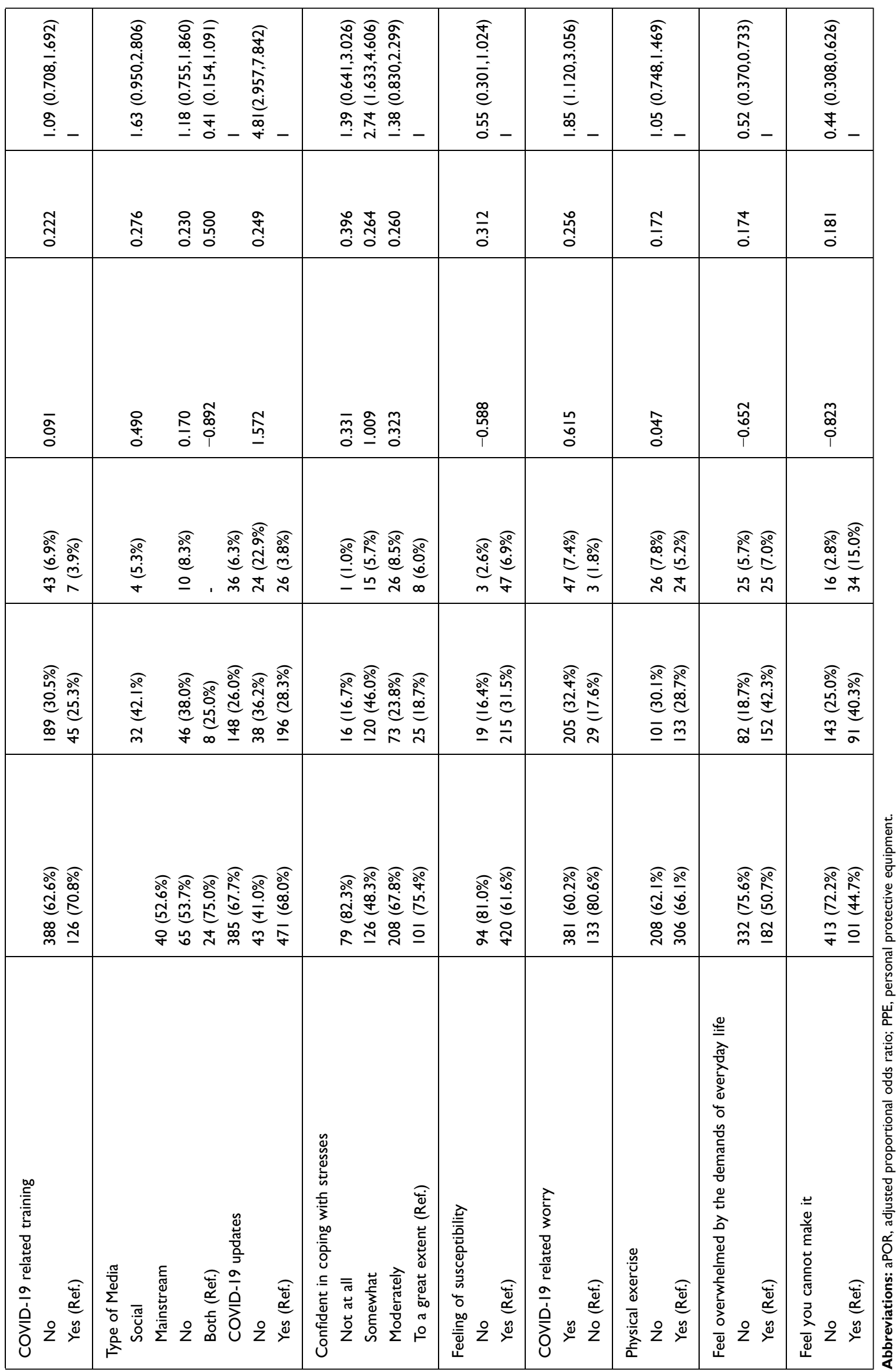


classify clinical severity of anxiety and large sample size covering a larger area.

\section{Conclusion}

The study revealed that the prevalence of anxiety disorder among health care workers was high in the study area. COVID-19 updates, contact with confirmed or suspected cases, confidence in coping with stresses, COVID-19 related worry, feel overwhelmed by the demands of everyday life, and feeling cannot make it were significant factors associated with the higher order of anxiety. The findings of the current study suggest immediate psychological intervention for health care workers in the study area is vital. Therefore, proactive measures should be taken by the stakeholders at different hierarchies to promote the psychological wellbeing of HCWs in order to control the impact of the Pandemic on the HCWs, and containing the pandemic.

\section{Acknowledgments}

The authors are grateful for the data collectors, the study participants, and supervisors for their co-operation during data collection. Our thanks also goes to Arba Minch University for funding and providing ethical clearance.

\section{Author Contributions}

All authors made substantial contributions to conception and design, acquisition of data, or analysis and interpretation of data; took part in drafting the article or revising it critically for important intellectual content; agreed to submit to the current journal; gave final approval of the version to be published; and agree to be accountable for all aspects of the work.

\section{Disclosure}

The authors report no conflicts of interest in this work.

\section{References}

1. Chirico F, Nucera G. Tribute to healthcare operators threatened by COVID-19 pandemic. J Health Soc Sci. 2020;5(2):165-168. doi:10.19204/2020/trbt1

2. Thseen N. Adjustment process during epidemics of COVID-19 and mental health. Int $J$ Creative Research Thoughts. 2020;8(7): 729-736.

3. Tandon R. The COVID-19 pandemic, personal reflections on editorial responsibility. Asian J Psychiatr. 2020;50:102100. doi:10.1016/j. ajp. 2020.102100

4. National Institute for Health and Care Excellence. Anxiety disorders. Quality standard; 2014. Available from: https://www.nice.org.uk/gui dance/qs53/resources/anxiety-disorders-pdf-2098725496261. Accessed August 2020.
5. McEvoy PM, Grove R, Slade T. Epidemiology of anxiety disorders in the Australian general population: findings of the 2007 Australian National survey of mental health and Wellbeing. Australian New Zealand J Psychiatry. 2011;45(11):957-967. doi:10.3109/ 00048674.2011.624083

6. Rana W, Mukhtar S, Mukhtar S. Mental health of medical workers in Pakistan during the pandemic COVID-19 outbreak. Asian J Psychiatr. 2020;51:102080. doi:10.1016/j.ajp.2020.102080

7. Liu X, Kakade M, Fuller CJ, et al. Depression after exposure to stressful events: lessons learned from the severe acute respiratory syndrome epidemic. Compr Psychiatry. 2012;53:15-23.

8. McAlonan GM, et al. Immediate and sustained psychological impact of an emerging infectious disease outbreak on health care workers. Can J Psychiatry. 2007;52:241-247. doi:10.1177/ 070674370705200406

9. Li L, Wan C, Ding R, et al. Mental distress among liberian medical staff working at the china ebola treatment unit: a cross sectional study. Health Qual Life Outcomes. 2015;13(1):1-6. doi:10.1186/ s12955-015-0341-2

10. Koh D, Lim MK, Chia SE, et al. Risk perception and impact of Severe Acute Respiratory Syndrome (SARS) on work and personal lives of healthcare workers in singapore what can we learn? Med Care. 2005;1:676-682. doi:10.1097/01.mlr.0000167181.36 730.cc

11. Chirico F, Nucera G, Magnavita N. Protecting the mental health of healthcare workers during the COVID-19 emergency. BJ Psych International. 2020;1-6. doi:10.1192/bji.2020.39

12. Lu YC, Chang YY, Shu BC. Mental symptoms in different health professionals during the SARS attack: A follow-up study. Psychiatric Quarterly. 2009;80(2):107. doi:10.1007/s11126-009-9095-5

13. Wu P, Fang Y, Guan Z, et al. The psychological impact of the SARS epidemic on hospital employees in China: exposure, risk perception, and altruistic acceptance of risk. Canadian J Psychiatry. 2009;54 (5):302-311. doi:10.1177/070674370905400504

14. Liu CY, Yang YZ, Zhang XM, et al. The prevalence and influencing factors in anxiety in medical workers fighting COVID-19 in China: a cross-sectional survey. Epidemiol Infect. 2020;Jan:1-7. doi:10.1017/ S0007485320000413

15. Zhang, et al. Mental health and psychosocial problems of medical health workers during the COVID-19 Epidemic in China. Psychother Psychosom Clinical Note. 2020. doi:10.1159/000507639

16. Vijay K, Sharma MD et al. Psychological impact of the COVID-19 pandemic on health care workers in Singapore. Ann Intern Med. doi:10.7326/M20-1083

17. Cheong D, Lee C. Impact of severe acute respiratory syndrome on anxiety levels of front-line health care workers. Hong Kong Med J. 2004;10(5):325.

18. Ethiopian monitor. Available from: https://ethiopianmonitor.com/ 2020/05/14/depression-anxiety-increase-three-fold-in-ethiopia-aftercovid-19/. Accessed October 28, 2020.

19. Spitzer RL, Kroenke K, Williams Williams JBW, Löwe B. A brief measure for assessing generalized anxiety disorder. Arch Intern Med. 2006;166:1092-1097. doi:10.1001/archinte.166.10.1092

20. Lai J, Ma S, Wang Y, et al. Factors associated with mental health outcomes among health care workers exposed to coronavirus disease 2019. JAMA Network Open. 2020;3(3):e203976. doi:10.1001/ jamanetworkopen.2020.3976

21. Elbay RY, Kurtulmuş A, Arpacioğlu S, Karadere E. Depression, anxiety, stress levels of physicians and associated factors in covid-19 pandemics. Psychiatry Res. 2020;27:113130. doi:10.1016/ j.psychres.2020.113130

22. Pappa S, Ntella V, Giannakas T, Giannakoulis VG, Papoutsi E, Katsaounou P. Prevalence of depression, anxiety, and insomnia among healthcare workers during the COVID-19 pandemic: A systematic review and meta-analysis. Brain Behav Immun. 2020;88:901-907. doi:10.1016/j.bbi.2020.05.026 
23. Zhu J, Sun L, Zhang L, et al. Prevalence and influencing factors of anxiety and depression symptoms in the first-line medical staff fighting against COVID-19 in Gansu. Front Psychiatry. 2020;11:386. doi: $10.3389 /$ fpsyt.2020.00386

24. Chew NW, Lee GK, Tan BY, et al. A multinational, multicentre study on the psychological outcomes and associated physical symptoms amongst healthcare workers during COVID-19 outbreak. Brain Behav Immun. 2020;88:559-565. doi:10.1016/j.bbi.2020.04.049

25. Tan BY, Chew NW, Lee GK, et al. Psychological impact of the COVID-19 pandemic on health care workers in Singapore. Ann Intern Med. 2020;6.

26. Chirico F, Magnavita N. Covid-19 infection in Italy: an occupational injury. S Afr Med J. 2020. Published online https://doi.org/10.7196/ SAMJ.2020.v110i6.14855.
27. Chirico F, Magnavita N. The crucial role of occupational health surveillance for health-care workers during the covid-19 pandemic. Workplace Health Saf. 2020;2165079920950161. doi:10.1177/ 2165079920950161

28. Wang C, Pan R, Wan X, et al. Immediate psychological responses and associated factors during the initial stage of the 2019 coronavirus disease (COVID-19) epidemic among the general population in China. Int $J$ Environ Res Public Health. 2020;17(5):1729. doi:10.3390/ijerph17051729

29. Barzilay R, Moore TM, Greenberg DM, et al. Resilience, COVID-19related stress, anxiety and depression during the pandemic in a large population enriched for healthcare providers. Transl Psychiatry. 2020;10(1):1-8. doi:10.1038/s41398-020-00982-4

\section{Publish your work in this journal}

Psychology Research and Behavior Management is an international, peer-reviewed, open access journal focusing on the science of psychology and its application in behavior management to develop improved outcomes in the clinical, educational, sports and business arenas. Specific topics covered in the journal include: Neuroscience, memory and decision making; Behavior modification and management; Clinical applications; Business and sports performance management; Social and developmental studies; Animal studies. The manuscript management system is completely online and includes a very quick and fair peer-review system, which is all easy to use. Visit http://www. dovepress.com/testimonials.php to read real quotes from published authors. 